\title{
Non-extremal Kerr black holes as particle accelerators
}

\author{
Sijie Gao*and Changchun Zhong ${ }^{\dagger}$ \\ Department of Physics, Beijing Normal University, \\ Beijing 100875, China
}

June 2, 2021

\begin{abstract}
It has been shown that extremal Kerr black holes can be used as particle accelerators and arbitrarily high energy may be obtained near the event horizon. We study particle collisions near the event horizon (outer horizon) and Cauchy horizon (inner horizon) of a non-extremal Kerr black hole. Firstly, we provide a general proof showing that particles cannot collide with arbitrarily high energies at the outter horizon. Secondly, we show that ultraenergetic collisions can occur near the inner horizon of a Kerr black hole with any spin parameter $a$.
\end{abstract}

PACS numbers: 04.70.Bw, 97.60.Lf

\section{Introduction}

Whether Kerr black holes can serve as particle accelerators with infinite collision energy has recently been discussed. Bañados, Silk and West [1] showed that particles falling from rest outside an extremal Kerr black hole $(a=M)$ can collide with arbitrarily high center of mass energies when the collision occurs arbitrarily close to the horizon. The BSW mechanism has been further discussed and generalized to different spacetimes (See e.g. 2]-7] ). Jacobson and Sotiriou [8] pointed out that infinite energies for the colliding particles can only be attained at infinite time. Authors also argued [9] that such a high energy may not be realizable due to the theoretical upper bound $a / M=0.998$ 10. The BSW mechanism would be more realistic if it worked for non-extremal Kerr black holes $(a<M)$. Although numerical analysis indicates that the collision energies are finite near the horizon of a non-extremal Kerr black hole, no rigorous proof has been given. In this paper, we prove analytically that infinite center of mass

\footnotetext{
*Email: sijie@bnu.edu.cn

$\dagger^{\dagger}$ Email: cczhong@mail.bnu.edu.cn
} 
energies can never be attained outside a non-extremal Kerr black holes. Compared with previous literature, our proof is general in the following senses. Firstly, the collision takes place anywhere outside the black hole, not confined to the equatorial plane $\theta=\frac{\pi}{2}$. Secondly, we allow the 4velocities of the two particles to be arbitrary. In particular, the 4 -velocities have non-vanishing $\dot{\theta}$ components. Finally, we release the restriction that particles fall from infinity, allowing them to fall from anywhere outside the black hole. Our analysis shows that infinite energies can only be attained at the horizon and one of the particles must have a critical angular momentum. However, with such an angular momentum, there always exists a potential barrier outside the black hole preventing the particle from approaching the event horizon.

Since the event horizon of a non-extremal Kerr black hole cannot serve as a particle accelerator creating infinite collision energies, it is natural to ask whether the inner horizon can make a difference. This issue has recently been explored by Lake [11. The author claimed in the original version that the center of mass energy for two colliding particles is generically divergent at the inner horizon and no fine tuning is required. Then in the Erratum this claim was withdrawn because physical constraints forbid such collisions. We reexamine this issue in details and arrive at the following conclusions. We first confirm, using different arguments, that a generic divergence at the inner horizon is not possible. We further show that for a critical angular momentum, the center of mass energy diverges at the inner horizon. Such a divergence is similar to that proposed by BSW where a critical angular momentum also is required. The difference is that there is no restriction on the spin parameter $a$. So in principle, infinite collision energies can be obtained near the inner horizon of any non-extremal Kerr black hole.

\section{Collisions near the event horizon $r=r_{+}$}

In this section, we consider two particles colliding outside a Kerr black hole and show that in any case, they cannot collide with arbitrarily high center of mass energies. The Kerr metric is given by 12 ]

$$
\begin{aligned}
d s^{2} & =-\left(\frac{\Delta-a^{2} \sin ^{2} \theta}{\Sigma}\right) d t^{2}-\frac{2 a \sin ^{2} \theta\left(r^{2}+a^{2}-\Delta\right)}{\Sigma} d t d \phi \\
& +\left(\frac{\left(r^{2}+a^{2}\right)^{2}-\Delta a^{2} \sin ^{2} \theta}{\Sigma}\right) \sin ^{2} \theta d \phi^{2}+\frac{\Sigma}{\Delta} d r^{2}+\Sigma d \theta^{2},
\end{aligned}
$$

where

$$
\begin{aligned}
\Sigma & =r^{2}+a^{2} \cos ^{2} \theta \\
\Delta & =r^{2}+a^{2}-2 M r .
\end{aligned}
$$


Without loss of generality, we shall choose

$$
M=1
$$

in the rest of this paper. We shall deal with the non-extremal case, i.e.,

$$
0<a<1 .
$$

Suppose a particle of mass $m$ moves in the spacetime with 4 -velocity $u^{a}=$ $\left(\frac{\partial}{\partial \tau}\right)^{a}$, where $\tau$ is the proper time. The 4 -velocity of one of the particle at the point of collision takes the general form

$$
u^{a}=\dot{t}\left(\frac{\partial}{\partial t}\right)^{a}+\dot{r}\left(\frac{\partial}{\partial r}\right)^{a}+\dot{\theta}\left(\frac{\partial}{\partial \theta}\right)^{a}+\dot{\phi}\left(\frac{\partial}{\partial \phi}\right)^{a} .
$$

The geodesic motion is determined by the following conserved quantities 12

$$
\begin{aligned}
E & =-g_{a b} u^{a}\left(\frac{\partial}{\partial t}\right)^{b}=\left(1-\frac{2 r}{\Sigma}\right) \dot{t}+\frac{2 a r \sin ^{2} \theta}{\Sigma} \dot{\phi} \\
L & =g_{a b} u^{a}\left(\frac{\partial}{\partial \phi}\right)^{b}=-\frac{2 a r \sin ^{2} \theta}{\Sigma} \dot{t}+\frac{\left(r^{2}+a^{2}\right)^{2}-\Delta a^{2} \sin ^{2} \theta}{\Sigma} \sin ^{2} \theta \dot{\phi} \\
-1 & =g_{a b} u^{a} u^{b}
\end{aligned}
$$

where $E$ is the conserved energy per unit mass and $L$ is the angular momentum per unit mass. Solving Eqs. (6) and (7) yields

$$
\begin{aligned}
\dot{t} & =\frac{a^{4} E-4 a L r+2 E r^{4}+a^{2} E r(2+3 r)+a^{2} E \Delta \cos 2 \theta}{2 \Delta \Sigma}, \\
\dot{\phi} & =\frac{a(2 E r-a L)+L \Delta \csc ^{2} \theta}{\Delta \Sigma} .
\end{aligned}
$$

Since $u^{a}$ is a future-directed timelike vector, it follows that $\dot{t}>0$ near the horizon $r=r_{+}$[12]. By expanding the numerator of Eq. (9) around $r=r_{+}$ and requiring the leading term to be non-negative, we find

$$
L \leq \frac{2 E}{a}\left(1+\sqrt{1-a^{2}}\right) .
$$

Note that Eq. (11) was also derived in [13] from the null energy condition.

Then Eq. (8) yields

$$
\dot{r}=-\frac{1}{2 \sqrt{2} \Sigma} \sqrt{S}
$$

where

$$
\begin{aligned}
S & =8 \Delta \Sigma\left(-1-\dot{\theta}^{2} \Sigma\right)-\csc ^{2} \theta\left[-a^{4} E^{2}+4 a^{2} L^{2}-6 a^{2} E^{2} r+16 a E L r\right. \\
& -16 L^{2} r-5 a^{2} E^{2} r+8 L^{2} r^{2}-4 E^{2} r^{4}+a^{2} E^{2} \Delta \cos 4 \theta \\
& \left.+4\left(-4 a E L r+E^{2} r^{4}+a^{2} L^{2}+a^{2} E^{2} r(2+r)\right) \cos 2 \theta\right]
\end{aligned}
$$


Note that we have chosen the minus sign for $\dot{r}$, referring to ingoing geodesics. We shall discuss the plus sign at the end of this section.

Obviously, a physically allowed trajectory satisfies

$$
S \geq 0 .
$$

Suppose two particles with the same mass $m$ collide each other. The center of mass energy is given by [1]

$$
E_{c . m .}=m \sqrt{2} \sqrt{1-g_{a b} u^{a} u_{2}^{b}} .
$$

For simplicity, define the effective center of mass energy

$$
E_{e f f}=-g_{a b} u^{a} u_{2}^{b} .
$$

We find

$$
E_{e f f}=-\Sigma \dot{\theta} \dot{\theta}_{2}-\frac{E^{\prime}}{8 \Delta \Sigma},
$$

where

$$
\begin{aligned}
E^{\prime} & =\left(-a^{4} E E_{2}+4 a^{2} L L_{2}-6 a^{2} E E_{2} r+8 a E_{2} L r+8 a E L_{2} r-16 L L_{2} r\right. \\
& -5 a^{2} E E_{2} r^{2}+8 L L_{2} r^{2}-4 E E_{2} r^{4}+4\left(-2 a\left(E_{2} L+E L_{2}\right) r+E E 2 r^{4}\right. \\
& \left.\left.+a^{2}\left(L L_{2}+E E_{2} r(2+r)\right)\right) \cos 2 \theta+a^{2} E E_{2} \Delta \cos 4 \theta\right) \csc ^{2} \theta \\
& +\sqrt{S} \sqrt{S_{2}} .
\end{aligned}
$$

Here $S_{2}$ is obtained by replacing $L, E, \dot{\theta}$ with $L_{2}, E_{2}, \dot{\theta}_{2}$ in Eq. (13).

Our purpose is to examine whether an infinite $E_{\text {eff }}$ defined in Eq. (17) exists under the constraints (44),(11), (14). As physical requiremens, all the constants $E, L$, etc. should be finite. Otherwise, infinite collision energies can be produced even in Minkowski spacetime. It is also reasonable to assume that $\dot{\theta}$ and $\dot{\theta}_{2}$ are finite at the horizon. This is because one can define

$$
L_{\theta} \equiv g_{a b} u^{a}\left(\frac{\partial}{\partial \theta}\right)^{b}=\Sigma \dot{\theta}
$$

as the "angular momentum with respect to $\theta$ ". Note that unlike $L, L_{\theta}$ is not constant along geodesics. However, it is plausible to require that $L_{\theta}$ be finite everywhere, particularly at the horizon. It then follows from Eq. (19) that $\dot{\theta}$ is finite at the horizon.

Hence, it is easy to see from Eq. (17) that an infinite $E_{\text {eff }}$ cannot be obtained unless $\Delta=0$, i.e., the collision must occur at the outer horizon $r=r_{+}$or the inner horizon $r=r_{-}$. To check if $E_{\text {eff }}$ could be infinite, we expand $E^{\prime}$ at $r=r_{+}$and find

$$
E^{\prime}=\alpha_{0}+\alpha_{1}\left(r-r_{+}\right)+\ldots,
$$


where

$$
\begin{aligned}
\alpha_{0} & =8\left[-a^{2} L L_{2}+E\left(-8+4 a^{2}-8 \sqrt{1-a^{2}}\right) E_{2}+2 a\left(E L_{2}+E_{2} L\right)\left(1+\sqrt{1-a^{2}}\right)\right. \\
& \left.+a^{2} \sqrt{\left(L-\frac{2 E\left(1+\sqrt{1-a^{2}}\right)}{a}\right)^{2}} \sqrt{\left(L_{2}-\frac{2 E_{2}\left(1+\sqrt{1-a^{2}}\right)}{a}\right)^{2}}\right] . \quad(21)
\end{aligned}
$$

Using Eq. (11), the square root terms can be simplified and one finds

$$
\alpha_{0}=0 \text {. }
$$

The vanishing of $\alpha_{0}$ is important because it rules out the divergence of $E_{c . m}$. for generic angular momentums.

Now it is obvious that $E_{\text {eff }}$ cannot be infinite unless $\alpha_{1}$ is infinite. Since $\left.\sqrt{S}\right|_{r=r_{+}}$appears in the denominator of $\alpha_{1}$, an infinite $\alpha_{1}$ requires

$$
\left.S\right|_{r=r_{+}}=2 a^{2}\left(L-L_{c}\right)^{2}=0 .
$$

Thus, if we choose

$$
L=L_{c}=\frac{2 E\left(1+\sqrt{1-a^{2}}\right)}{a},
$$

$E_{c . m}$. will become arbitrarily large at the horizon $r=r_{+}$. However, to make sure that the particle with this critical angular momentum can actually reach the horizon, Eq. (14) must hold outside the horizon. By Taylor expansion, we find

$$
S=a_{0}+a_{1}\left(r-r_{+}\right)+\ldots
$$

For $L=L_{c}, a_{0}=0$ and $a_{1}$ is given by.

$$
a_{1}=b_{1}+\left(\frac{2 E^{2}}{a^{2} \sin ^{2} \theta}+2 \dot{\theta}^{2}\right) b_{2},
$$

where

$$
\begin{aligned}
b_{1} & =-32+32 a^{2}-32 \sqrt{1-a^{2}}+16 a^{2} \sqrt{1-a^{2}}-16 a^{2} \sqrt{1-a^{2}} \cos ^{2} \theta \\
b_{2} & =-64+80 a^{2}-16 a^{4}+\sqrt{1-a^{2}}\left(-64+48 a^{2}-3 a^{4}-a^{4} \cos 4 \theta\right) \\
& +4 a^{2}\left(-4+4 a^{2}-4 \sqrt{1-a^{2}}+4 a^{2} \sqrt{1-a^{2}}\right) \cos 2 \theta .
\end{aligned}
$$

Simple analysis shows that both $b_{1}$ and $b_{2}$ are negative for $0<a<1$. Therefore,

$$
a_{1}<0
$$




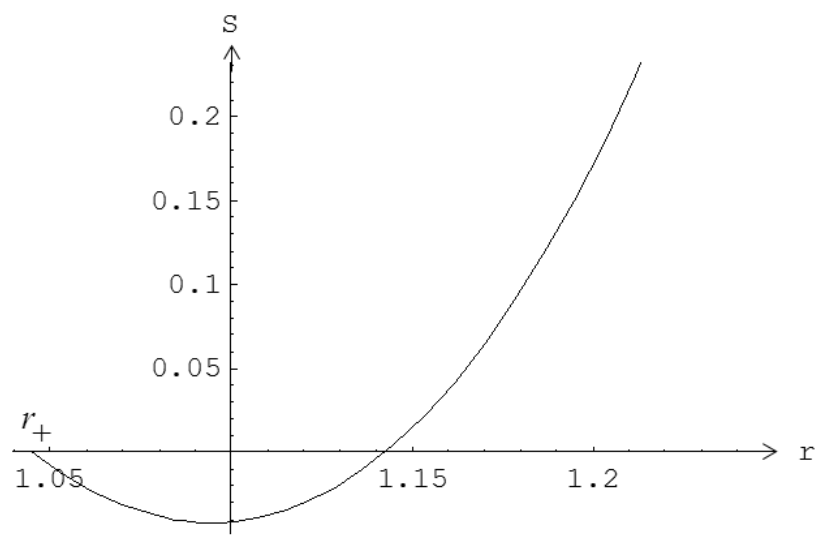

Figure 1: The plot of $S(r)$ in the region $r>r_{+}$. The parameters are chosen as: $a=0.999, \theta=\frac{\pi}{2}, L=L_{c}$.

which means $S$ is negative near the horizon and consequently the particle with $L=L_{c}$ cannot approach the horizon. The barrier outside the horizon is illustrated in Fig. 1].

In the above argument, we have chosen the minus sign in Eq. (12) for both particles. The same choice was made by BSW. What if the two particles take different signs? In that case, the plus sign in front of $\sqrt{S}$ in Eq. (18) will become a minus sign and consequently $\alpha_{0}$ in Eq. (20) will not vanish. Therefore, such two particles will collide with infinite energy even without a fine turning on angular momentum. But to make this happen, one of the particles must be outgoing $(\dot{r}>0)$ on the horizon. For a nonextremal black hole, even holding a particle still at the horizon requires an infinite local force 12. Therefore, the configuration with $\dot{r}>0$ should be ruled out.

\section{Collisions near the inner horizon $r=r_{-}$.}

Now we discuss the motions in the region $r_{-}<r<r_{+}$. Due to the different natures of the inner and outer horizons, some of the arguments in section 2 will break down in this section. We shall highlight the differences.

As illustrated in Fig. 2 we consider two particles crossing the event horizon of the right-hand universe and hitting each other at the Cauchy horizon of the left-hand universe. The general equations (5)-(10) remain unchanged. However, Eq. (11) is derived from the fact that $\nabla^{a} t$ is a pastdirected timelike vector, which is no longer true outside the inner horizon. Instead, $\nabla^{a} r$ becomes past-directed in this region and any particle must 


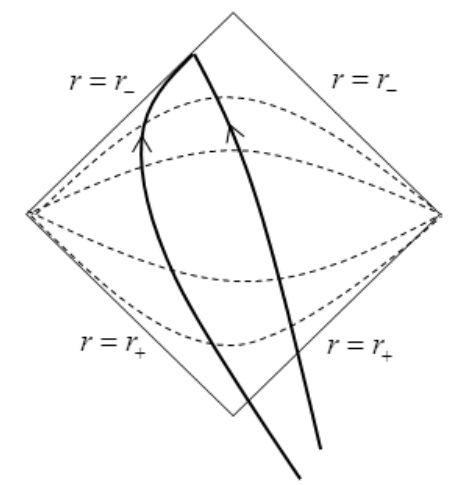

Figure 2: Penrose diagram for the Kerr spacetime in the region $r_{-} \leq r \leq$ $r_{+}$. Two particles cross the event horizon and collide at the inner horizon. The dashed lines refer to surfaces of constant $r$.

fall in the direction of $\dot{r}<0$, i.e.,

$$
\dot{r}=-\frac{1}{2 \sqrt{2} \Sigma} \sqrt{S}<0 .
$$

It should be noticed that Eq. (12) holds because we have chosen the ingoing mode for both particles in the region $r>r_{+}$, while Eq. (30) holds for any particle in the region $r_{-}<r<r_{+}$. Note that Eqs. (13)-(18) remain unchanged. Then we expand $E^{\prime}$ at $r=r_{-}$. Corresponding to Eq. (20), we have

$$
E^{\prime}=\alpha_{0}+\alpha_{1}\left(r-r_{-}\right)+\ldots
$$

where

$$
\begin{aligned}
\alpha_{0} & =8\left[-a^{2} L L_{2}+E\left(-8+4 a^{2}+8 \sqrt{1-a^{2}}\right) E_{2}+2 a\left(E L_{2}+E_{2} L\right)\left(1-\sqrt{1-a^{2}}\right)\right. \\
& \left.+a^{2} \sqrt{\left(L-\frac{2 E\left(1-\sqrt{1-a^{2}}\right)}{a}\right)^{2}} \sqrt{\left(L_{2}-\frac{2 E_{2}\left(1-\sqrt{1-a^{2}}\right)}{a}\right)^{2}}\right]
\end{aligned}
$$

This equation can be simplified by choosing the ingoing mode for both particles, as depicted in Fig. 2. Here in the region $r_{-}<r<r_{+}$, the ingoing mode means $\dot{t}<0$ (By the same argument as given at the end of section 2, $\dot{t}>0$ at the left-hand Cauchy horizon is not physically realizable). Then using the same argument which led to Eq. (11), we find

$$
\left(L-\frac{2 E\left(1-\sqrt{1-a^{2}}\right)}{a}\right)\left(L_{2}-\frac{2 E_{2}\left(1-\sqrt{1-a^{2}}\right)}{a}\right) \geq 0,
$$


which leads to

$$
\alpha_{0}=0 \text {. }
$$

Again, the necessary condition for $E_{\text {eff }}$ blowing up at the horizon is that $\alpha_{1}$ blows up, which requires

$$
\left.S\right|_{r=r_{-}}=8 a^{2}\left(L-L_{c}^{\prime}\right)^{2}=0,
$$

where

$$
L_{c}^{\prime}=\frac{2 E\left(1-\sqrt{1-a^{2}}\right)}{a} .
$$

Therefore, an infinite collision energy near the inner horizon requires that one of the particles has the momentum

$$
L=L_{c}^{\prime}
$$

and the other particle has any different angular momentum. For the collision near the event horizon, we have shown that a potential barrier always exists. But the geodesic motions near the inner horizon are very different. We shall show that there is no potential barrier in the vicinity of the inner horizon. For our purposes, it is sufficient to consider the motions in the equatorial plane and let $E=1$, in which case, $S$ given in Eq. (13) reduces to

$$
S=\frac{16 r\left[a^{4}-4\left(1-\sqrt{1-a^{2}}\right)(r-2)+a^{2}\left(r^{2}+2 r+4 \sqrt{1-a^{2}}-8\right)\right]}{a^{2}} .
$$

By Taylor expansion, we find

$$
S=\frac{32}{a^{2}}\left[5-a^{2}-3 \sqrt{1-a^{2}}-\frac{4\left(1-\sqrt{1-a^{2}}\right)}{a^{2}}\right]\left(r-r_{-}\right)+\ldots
$$

By plotting the coefficient of $r-r_{-}$as a function of $a$, we see immediately that $S>0$ for any $0<a<1$ in the vicinity of $r=r_{-}$.

Now we show, by a concrete example, that such a collision can be realized at the Cauchy horizon for two particles falling from rest at infinity.

The orbits of the two particles are confined to the equatorial plane $\theta=\frac{\pi}{2}$. The parameters are chosen as

$$
\begin{aligned}
E & =E_{2}=1 \\
L & =L_{c}^{\prime} \\
L_{2} & =\frac{L_{c}^{\prime}}{2} .
\end{aligned}
$$

As depicted in Fig. 3, the center of mass energy blows up at $r=r_{-}$. Fig. 4 shows $S>0$ and $S_{2}>0$ for all $r>r_{-}$, i.e., the two particles can 


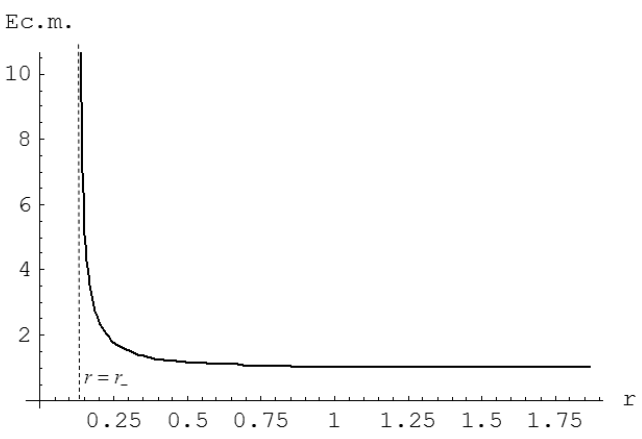

Figure 3: Plot of the center-of-mass energy
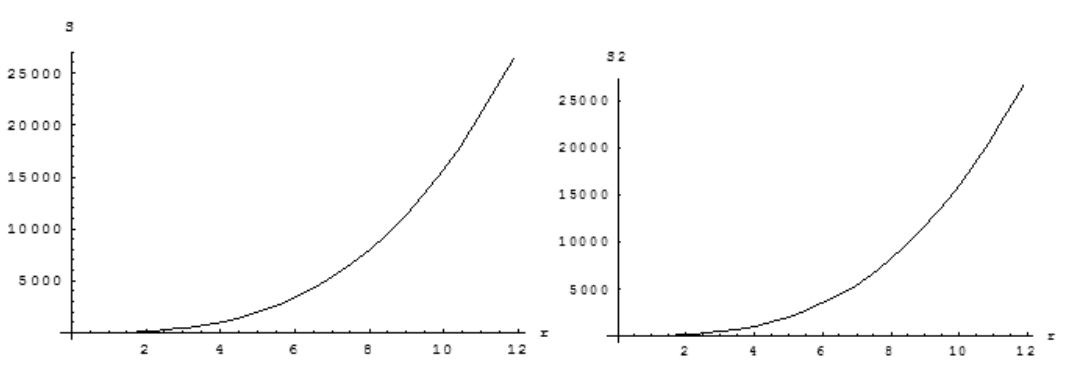

Figure 4: Plots of the functions $S(r)$ and $S_{2}(r)$. Both functions are positive in the range $r>r_{-}$.

fall from infinity all the way to the Cauchy horizon. Fig. 5 plots $\dot{t}(r)$ in the region $r>r_{-}$. We see that $\dot{t}$ and $\dot{t}_{2}$ are positive in the region $r>r_{+}$, as expected. For $r_{-}<r<r_{+}, \dot{t}$ and $\dot{t}_{2}$ are negative. This means both particles must hit the left-hand Cauchy horizon, as depicted in Fig. 2. Otherwise, there will be a turning point $\dot{t}=0$ in this region. It should also be noticed that $\dot{t}_{2} \rightarrow-\infty$, meaning that this particle will cross the Cauchy horizon. But for the first particle which has a critical angular momentum, $\dot{t}$ is finite as $r \rightarrow r_{-}$. Thus, instead of crossing the Cauchy horizon, this particle spirals asymptotically onto the horizon. This is the necessary mechanism for an infinite center of mass energy as pointed out in [8]. One can also check that $\dot{r}$ vanishes on the Cauchy horizon while $\dot{r}_{2}$ does not, which is consistent with the behaviors of $\dot{t}$ and $\dot{t}_{2}$.

\section{Conclusions}

We have provided a rigorous proof showing that the center of mass energy cannot be divergent at the event horizon of a non-extremal black hole. This 


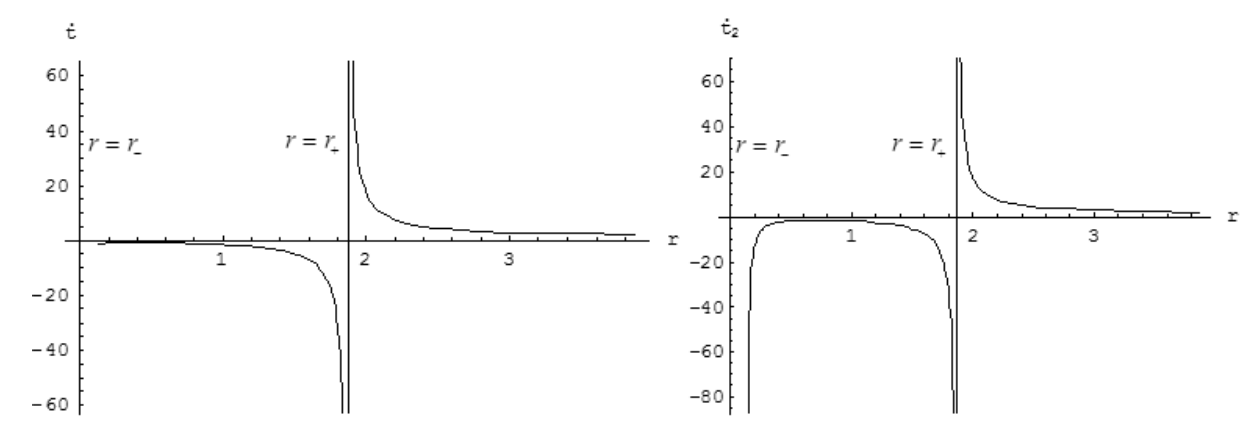

Figure 5: Plots of $\dot{t}(r)$ and $\dot{t}_{2}(r)$.

proof is general and exhausts all of the possibilities. The motion of particles is not confined to the equatorial plane and the particles may be released from any point outside the black hole. We find that a critical angular momentum is required for the divergence of $E_{c . m}$. at the horizon. However, with this angular momentum, the particle can never reach the horizon. In relation to Lake's work, we first prove that no divergence of energy occur at the inner horizon for particles with generic angular momentums. A critical angular momentum is required for the divergence. We show with an explicit example that two particles can fall from infinity all the way to the inner horizon and collide with an arbitrarily high center of mass energy. We have shown that such arbitrarily high energies can be obtained in any non-extremal Kerr spacetime, unlike the case in [1] that requires the black hole to be exactly extremal. Since extremal black holes do not exist in nature, the ultraenergetic collisions near Cauchy horizons may be more practical and realizable.

\section{Acknowledgements}

This research was supported by NSFC grants 10605006, 10975016 and by "the Fundamental Research Funds for the Central Universities".

\section{References}

[1] M.Bañados, J.Silk and S.M.West, Phys.Rev.Lett. 103, 111102 (2009).

[2] O.B.Zaslavskii, Phys. Rev. D 82, 083004 (2010).

[3] O.B.Zaslavskii, Class.Quant.Grav. 28105010 (2011) .

[4] S.W. Wei, Y.X. Liu, H.Guo, Chun-E Fu, Phys.Rev. D 82103005 (2010).

[5] S.W. Wei, Y.X. Liu, H.Guo, Chun-E Fu, JHEP 1012 (2010) 066. 
[6] M.Kimura, K.Nakao, H.Tagoshi, Phys.Rev. D 83044013 (2011).

[7] T.Harada, M.Kimura, Phys.Rev.D 83084041 (2011).

[8] T.Jacobson and T.P.Sotiriou, Phys. Rev.Lett. 104, 021101 (2010).

[9] E.Berti, V.Cardoso, L.Gualtieri, F.Pretorius, and U.Sperhake, Phys.Rev.Lett. 103, 239001 (2009).

[10] K.S.Thorne, Astrophys.J. 191,507(1974).

[11] K.Lake, Phys. Rev. Lett. 104, 211102(2010) [Erratum-ibid. 104, 259903 (2010)].

[12] R.M. Wald, General Relativity, The University of Chicago Press, (Chicago, 1984).

[13] T.Jacobson and T.P.Sotiriou, Phys. Rev.Lett. 103, 141101 (2009). 